\title{
O uso do jogo Robocode para desenvolvimento de carreiras em STEM e habilidades do século XXI: um estudo de caso nacional
}

\author{
Gabriel Coutinho Natucci ${ }^{1}$, Jhasmani Tito Cruz ${ }^{1}$, Marcos A. F. Borges ${ }^{1}$, Regina Moraes ${ }^{1}$ \\ ${ }^{1}$ Faculdade de Tecnologia - Universidade Estadual de Campinas (UNICAMP) \\ Caixa Postal 456 - 13484-332 - Limeira - SP - Brazil \\ \{gabrielnatucci, jhasmaniectt\}@gmail.com,\{marcosborges, regina\}@ft.unicamp.br
}

\begin{abstract}
Universities and traditional learning approaches have been challenged in educating future professionals in competences such as computational thinking, creativity and other 21 st-century skills. This work proposes to analyse a pedagogical approach that enables the learning of these skills by studying an application of the serious game Robocode in a competitive national tournament. It was possible to conclude that this approach fosters computational thinking and other skills like creativity and team collaboration, therefore being a viable option for better preparing students for technology jobs.
\end{abstract}

Resumo. Universidades e práticas de ensino tradicionais têm enfrentado desafios ao preparar futuros profissionais em competências como o pensamento computacional, trabalho em equipe e outras habilidades do século XXI. Este trabalho tem por objetivo explorar novas práticas pedagógicas, analisando o uso do jogo sério Robocode dentro de um ambiente competitivo e como essa prática contribui para o desenvolvimento dessas competências. Foi possível verificar que essa prática estimula competências de pensamento computacional e habilidades do século XXI, sendo uma opção lúdica para a formação de profissionais mais preparados para o trabalho futuro com tecnologia.

\section{Introdução}

A crescente demanda por profissionais em STEM (Ciência, Tecnologia, Engenharia e Matemática) é uma tendência mundial, oriunda da revolução que áreas como a inteligência artificial e Indústria 4.0 vem trazendo para as profissões e a sociedade como um todo. Novos profissionais ligados ao uso / criação de tecnologia são cada vez mais importantes e são considerados como os mais significativos para o mercado técnico do futuro [World Economic Forum 2018]. Entretanto, atender a essa demanda utilizando-se do ensino tradicional esbarra na escassez de educadores com formação em STEM. As escolas e universidades tem enfrentado desafios ao preparar alunos que optem por uma carreira profissional nessas áreas, considerando fatores como a própria percepção do aluno em relação a suas habilidades em STEM [Alhaddab and Alnatheer 2015].

Mesmo estudantes que possuem um amplo domínio técnico precisam adquirir outras habilidades além das apresentadas no ensino tradicional, sendo encorajados a pensar de forma crítica, a resolver problemas complexos, a trabalhar em equipe e a praticar ações de liderança, um novo conjunto de habilidades conhecido como Habilidades do Século XXI (HS21) [Shute et al. 2017]. Dentre essas novas habilidades, destaca-se a necessidade de pensar criticamente através de novas tecnologias - o Pensamento Computacional (PC). 
PC é uma "abordagem para resolver problemas, projetar sistemas e entender comportamentos humanos que se baseia em conceitos da ciência da computação" [Wing 2006], usando técnicas de programação, sem se limitar a esse aspecto técnico [Wing 2008]. O PC se aplica tanto a resolução de problemas bem estruturados, quanto aqueles do mundo real, repletos de incertezas e nos quais soluções nem sempre são definitivas ou facilmente desenvolvidas [Shute et al. 2017]. O ensino tradicional não tem conseguido apoiar o desenvolvimento dessas habilidades no volume e profundidade necessários, principalmente quando aplicado às geracões mais jovens, que procuram informação rápida e a imediata aplicação do aprendizado [Seemiller and Grace 2017]. Os mais jovens ainda tem como objetivo a rápida compreensão de conceitos, principalmente utilizando os meios digitais [Shatto and Erwin 2016]. Assim, é necessário estimular novas práticas educativas que trabalhem essas três questões de forma motivadora e adequada ao perfil dos jovens.

Entre as novas ferramentas e práticas que podem ser usadas para enfrentar esses desafios estão o uso de jogos digitais e o aprendizado baseado em problemas (problem-based learning - PBL). A aplicação de jogos na educação está associada a uma série de benefícios, tais como uma maior efetividade no aprendizado em geral [Sousa and Costa 2018] além da melhoria de certas capacidades cognitivas, tais como distribuição de atenção e mudanças comportamentais positivas [Boyle et al. 2016]. O PBL é uma abordagem pedagógica que permite que alunos aprendam pelo engajamento com problemas reais [Yew and Goh 2016]. Entre seus benefícios estão uma maior retenção de conhecimento por longos períodos e uma maior independência por parte do aluno em relação ao apoio do professor [Moust et al. 2016].

O Robocode é uma plataforma de programação em Java em que alunos aprendem a codificar robôs, devendo competir com outros oponentes ou robôs pré-programados de forma lúdica [Amaral et al. 2015]. O Robocode apresenta uma relação direta com PBL ao propor ao aluno a programação de um robô para competir e vencer. O jogo pode ser usado em uma prática pedagógica tradicional ou ser usado como uma forma de competição científica, com grupos de alunos desenvolvendo um único robô e competindo em torneios organizados por instituições e escolas [J. and Gibson 2006]. A proposta de competições científicas neste caso apresenta diversos benefícios, como a motivação e interesse dos participantes para continuar estudos em STEM [Ali et al. 2018], além de estimular a trabalharem em áreas relacionadas [Meira 2016]. Adicionalmente, o uso de robôs mesmo que "virtuais", apenas em tela, traz benefícios educacionais adicionais, tais como uma maior facilidade na abstração de algoritmos e solução de problemas e a apresentação de uma programação clara e objetiva, facilitando o uso de certas teorias pedagógicas, tais como o construtivismo [Weintrop and Wilensky 2012]. Embora a prática competitiva de Robocode tenha sido abordada na literatura, pouco se tem estudado sobre o impacto que a participação em torneios baseados em Robocode podem gerar, no médio e longo prazo, nos competidores, incluindo a aquisição de competências de PC, a influência em suas decisões de carreira ou mesmo o aprendizado de outras habilidades essenciais, como trabalho em equipe, gestão de projetos e inteligência emocional [World Economic Forum 2018].

O presente trabalho apresenta uma análise da aplicação do jogo sério Robocode em competições científicas inspiradas em PBL, visando identificar o impacto desta participação na compreensão de conceitos de PC, no desenvolvimento de habilidades do 
futuro e no impacto de carreiras em STEM. Considerou-se como foco estudantes e organizadores de torneios de Robocode do ensino médio e superior no âmbito nacional, em especial competições do torneio Robocode Brasil, coordenado pelo Laboratório de Informática, Aprendizado e Gestão (LIAG) da UNICAMP ${ }^{1}$. Esse torneio foi escolhido por ser o único no Brasil que não é limitado a uma única instituição de ensino, sendo que qualquer professor pode nele inscrever seus alunos.

O artigo está organizado da seguinte forma: a Seção 2 descreve em detalhes a metodologia de pesquisa utilizada para o estudo, bem como considerações sobre as HS21 analisadas e competências de PC observadas; a Seção 3 apresenta as observações e resultados obtidos a partir da análise das competições científicas, bem como discute essas observações; e a Seção 4 conclui o artigo, apresentando futuras direções de pesquisa.

\section{Metodologia}

Para analisar o impacto das competições científicas usando Robocode foi selecionado o método de análise de estudo de caso, definido como uma descrição abrangente de um fenômeno em particular, com o propósito de identificar relações entre variáveis de interesse ou analisar seu progresso/performance [Starman 2013]. Neste contexto, o fenômeno estudado são as competições científicas coordenadas através do torneio Robocode Brasil. Ele ocorre anualmente desde o ano de 2010, organizado pelo LIAG.

Esse evento é composto por duas fases: torneios locais e liga. Os torneios locais são competições de Robocode realizadas no âmbito de uma mesma instituição de ensino e a liga é composta por etapas organizadas com as equipes finalistas de cada torneio local. As regras específicas do evento estão disponibilizadas no site [LIAG - UNICAMP 2020]. As etapas e critérios de classificação dos torneios locais são definidos por cada instituição de ensino de forma independente. Se uma instituição não apresentar o número mínimo de participantes para a organização de um torneio local e tiver interesse em participar do torneio, poderá se inscrever na categoria de torneio público, competindo com outras equipes na mesma situação, seguindo os critérios de pontuação da liga.

Para o estudo deste torneio, optou-se por uma análise mista, envolvendo tanto aspectos qualitativos quanto quantitativos visando uma compreensão simultaneamente detalhada e abrangente do impacto desse fenômeno [Leydens et al. 2004]. Esse estudo foi realizado entre participantes e coordenadores de torneios locais e da liga nos anos de 2014 a 2019, tratando-se de uma análise retrospectiva, possibilitando avaliar a evolução da competição ao longo dos anos. Na análise dos participantes, optou-se por um estudo quantitativo utilizando-se a construção e distribuição de formulários de pesquisa (survey); as perguntas do formulário encontram-se disponíveis no link $<\mathrm{https} / / /$ bit.ly/2G9zH9A $>$. Esse método foi escolhido por permitir um maior alcance e representatividade dos alunos competidores [Almeida et al. 2017]. Já para a análise dos coordenadores e professores envolvidos na organização e divulgação dos torneios, optou-se por uma análise qualitativa na forma de entrevistas semi-estruturadas, com perguntas pré-definidas, porém dando ao entrevistador a liberdade para se aprofundar em certas respostas, caso julgasse necessário [Leydens et al. 2004]. Esse aspecto qualitativo foi escolhido pois permite trazer informações mais detalhadas e opiniões mais pessoais dos entrevistados sob o fenômeno de interesse [Almeida et al. 2017].

\footnotetext{
${ }^{1}$ https://liag.ft.unicamp.br/robocode/
} 
A análise dos participantes do torneio foi dividida em 3 aspectos: a influência das competições na escolha de carreira, o aprendizado e utilização de HS21/PC durante a competição e uma avaliação do Robocode em si, buscando analisar sua aplicação em um contexto de PBL na forma de competições. Elas buscaram inferir a capacidade do Robocode acrescentar algum conceito novo de computação, quais conhecimentos adquiridos no torneio foram utilizados no trabalho e/ou estudo do participante, se houve participação em outros torneios similares e se o participante indicaria o Robocode para algum amigo. Foi questionada ainda a experiência do participante, em escala Likert, com relação ao sistema de programação, bem como uma justificativa para a nota selecionada; foram avaliadas também em escala Likert quais estratégias de PC utilizadas foram usadas no torneio. Foram questionados também os conceitos não-técnicos mais utilizados, os aspectos importantes para o robô desenvolvido durante o torneio e se a participação na competição despertou algum interesse em carreiras futuras.

Quanto à competências de PC, embora exista pouco consenso em quais competências constituem o pensamento computacional [Brennan and Resnick 2012], foram avaliados os seguintes aspectos neste formulário: processamento e análise de dados, modelagem e simulação, algoritmos e programação, abstração e decomposição de problemas, automação e paralelismo [Shute et al. 2017].

Além dessas perguntas, foram coletadas informações básicas sobre o participante sem ferir sua privacidade, tais como o nível de escolaridade no momento da participação da competição. Os dados obtidos a partir deste formulário foram analisados graficamente usando a linguagem de programação Python através da plataforma Google Colab, bem como avaliados qualitativamente em respostas abertas. $\mathrm{O}$ formulário foi encaminhado por listas de email para 749 ex-participantes do Robocode Brasil; desses, 68 responderam o questionário solicitado, dos quais 9 participaram do torneio em mais de um ano, sendo contados múltiplas vezes nas análises. O questionário também foi divulgado através dos próprios coordenadores contatados, bem como divulgados em páginas de redes sociais do LIAG; entretanto, a forma mais eficiente de divulgação foi o contato direto através do email dos participantes.

Quanto aos coordenadores do torneio, foram contatados para entrevista aqueles que organizaram um ou mais torneios durante o período avaliado, listados a partir da base de ex-participantes. Vale ressaltar que para o torneio analisado, não é necessária a presença de um coordenador local, podendo os participantes competir sem coordenação local. As entrevistas foram realizadas em videoconferências usando a plataforma Google Meet, partindo das perguntas disponíveis no link $<$ https://bit.ly/2G9zH9A $>$. O foco da entrevista foi avaliar como foram organizados os torneios locais em cada instituição, além de obter impressões desses coordenadores a respeito do engajamento dos alunos e seu impacto no aprendizado. Ao todo, foram entrevistados 12 coordenadores locais dos 17 mapeados através dos dados históricos, sendo que desses últimos somente dois pertenciam a outros estados (Goiás e Bahia, respectivamente).

Finalmente, para fins de comparação, foi realizada uma entrevista qualitativa com coordenadores de um torneio local de Robocode que não participa da liga nem mantém qualquer relação com o evento Robocode Brasil, totalizando 13 entrevistas qualitativas, buscando identificar as diferenças na organização de competições científicas, aplicações dos conceitos de PBL e uso de jogos sérios na educação. 


\section{Resultados e Discussões}

Nesta seção são apresentados os resultados obtidos com o survey em que foram alvos os participantes do torneio Robocode, bem como os resultados obtidos junto aos coordenadores e professores envolvidos na organização e que participaram de entrevistas semi-estruturadas. A entrevista foi composta por perguntas pré-definidas, concedendo também flexibilidade ao entrevistador para que pudesse aprofundar em algum assunto mais relevante. A Subseção 3.1 apresenta os resultados do survey enquanto a Subseção 3.2 apresenta os resultados das entrevistas.

\subsection{Alunos}

Dos ex-participantes que responderam ao questionário, a maioria teve participação nos torneios de 2018 e 2019; em relação à escolaridade, no momento da participação os respondentes estavam divididos igualmente entre ensino médio e ensino superior. A Figura 1 ilustra em mais detalhes o perfil desses respondentes.

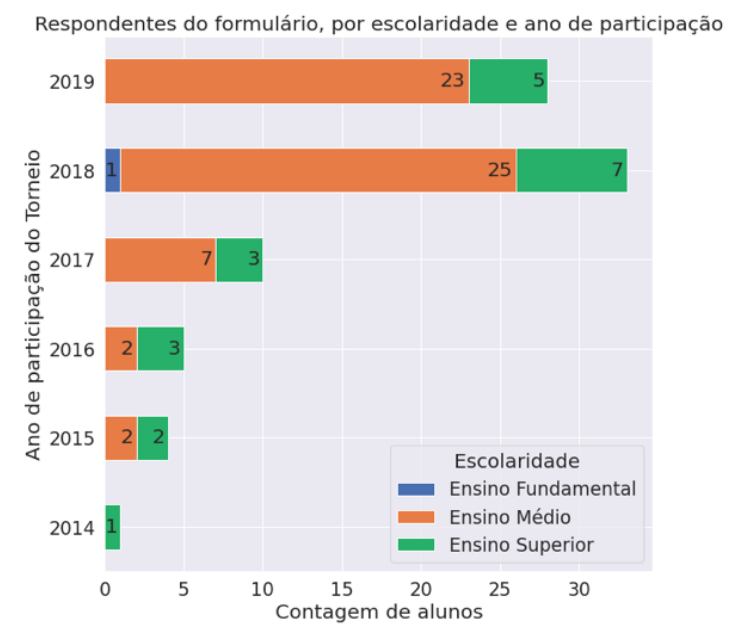

Figura 1. Perfil dos Alunos respondentes do formulário de pesquisa

Em relação à avaliação do torneio, cerca de $64,19 \%$ dos entrevistados também indicaram a intenção de participar novamente, porém apenas 9 desses de fato participaram em múltiplas competições. Essa intenção não realizada pode ser um indicativo de potenciais problemas com divulgação do torneio para ex-alunos, ou mesmo uma perda do interesse dos alunos em competições científicas à medida em que avançam nos cursos de formação superior. Essa última explicação foi confirmada com os coordenadores entrevistados, que indicaram um aumento das responsabilidades dos alunos ao longo do seu ensino, motivo pelo qual a maioria dos participantes dos torneios são aqueles estudantes de primeiros anos de ensino técnico ou superior.

Uma parcela significativa dos respondentes também mostrou incerteza em participações futuras, sendo estes $24,69 \%$ dos entrevistados. Dentre esses, a principal razão dada foi a falta de tempo, seguida da natureza do tipo do desafio proposto na competição. Entretanto, a maioria esmagadora indicaria a competição a um amigo $(86,41 \%)$. Dentre os principais motivos relatados desta indicação encontram-se o estímulo ao aprendizado de programação e a intenção de seguir uma carreira na área de computação 
IX Congresso Brasileiro de Informática na Educação (CBIE 2020)

Anais do XXXI Simpósio Brasileiro de Informática na Educação (SBIE 2020)

$(42,85 \%)$, por estimular o espírito lúdico $(23,37 \%)$ e por estimular o trabalho em equipe $(14,28 \%)$.

Observa-se portanto que o torneio estimula seus participantes a compreenderem melhor conceitos de programação e os estimulam a seguir uma carreira envolvendo STEM de forma lúdica e engajante. Neste contexto, $61,72 \%$ dos entrevistados reportaram um interesse em melhorar habilidades de programação. Esse interesse também foi observado pelos coordenadores do torneio, sendo que $53,84 \%$ desses verificaram um aumento da capacidade técnica dos alunos em conceitos de programação e abstrações relacionadas, tais como a orientação a objetos. Além disso, alguns coordenadores também apontaram o torneio como uma possibilidade dos alunos experientes em programação amadurecerem seus conceitos, bem como auxiliar e motivar alunos iniciantes a melhorar suas competências técnicas.

Outros interesses de carreira foram apresentados pelos estudantes: $39,51 \%$ reportaram interesse em exercer posições de coordenação e liderança após o torneio e 37,03\% dos alunos demonstraram interesse em seguir carreira na área de computação. Embora esses interesses não possam ser diretamente observados pelos coordenadores do torneio, $69,23 \%$ da coordenação reportou observar o trabalho em equipe dos participantes e $30,77 \%$ indicou um interesse dos participantes em desenvolver uma carreira em computação.

Em termos do uso da interface Robocode, atingiu-se a média de 3.73 com desvio de 0.99 considerando-se a escala Likert de 1 a 5 , indicando oportunidades de melhoria. A principal reclamação dos entrevistados foi a interface ultrapassada da plataforma $(29,63 \%)$, o que dificultava seu uso e comprometia a compreensão dos conceitos de programação. Três dos coordenadores também reportaram uma necessidade de atualizar a interface da plataforma, sendo que um deles sugeriu também atualizações na ferramenta. Um dos coordenadores apontou grande dificuldade no uso da plataforma, e sugeriu a possibilidade de aplicar interfaces de programação com blocos, como uma alternativa para aqueles que ainda não estão familiarizados com a programação. Outra sugestão de um coordenador se refere a uma maior abrangência de linguagens de programação além da mais utilizada na plataforma (Java). Segundo ele, o uso desta linguagem notoriamente descritiva pode desmotivar alunos a participarem da competição, sendo preferível o uso de linguagens mais populares e de mais alto nível, tal como Python. No entanto, é importante observar que a linguagem Java segue sendo a mais utilizada no mundo [ComputerWorld 2019].

Com relação às competências de $\mathrm{PC}$ usadas nas competições, a maioria dos participantes entrevistados $(74,07 \%)$ relata ter desenvolvido alguma competência. As mais utilizadas e desenvolvidas foram a análise de dados $(31,67 \%)$, seguidos por otimização e abstração do robô $(21,67 \%)$, além de algoritmos e programação $(21,67 \%)$. Além disso, $88,89 \%$ dos participantes declararam ter gostado de desenvolver a lógica de programação dos robôs em competição e 86,42\% declararam ter gostado de planejar e pensar em novas estratégias (resolução de problemas) durante a partida. Essa preferência dos competidores por desenvolver habilidades de resolução de problemas e abstração é um indicativo de que a competição analisada vai além do âmbito da prática de programação, estimulando participantes a trabalharem em soluções inventivas para avançarem no torneio. 
Considerando as HS21, as competências mais trabalhadas pelos participantes são a resolução de problemas $(83,95 \%)$, trabalho em equipe $(67,90 \%)$ e criatividade $(79,01 \%)$. Essas competências também foram as mais comentadas pelos coordenadores durante as entrevistas, sendo a criatividade e resolução de problemas observadas por $76,92 \%$ durante a organização do torneio. Destaca-se também o desenvolvimento de competências ligadas à gestão de projetos, observadas por 69,23\% dos coordenadores e trabalhadas pelos alunos para gerenciar os prazos das competições e atividades de cada integrante da equipe no desenvolvimento do robô.

Com base nos resultados, é possível concluir que o torneio estimula seus participantes a melhorarem suas competências de PC e os estimulam a trabalhar profissionalmente em áreas correlatas, desenvolvendo HS21 como a resolução de problemas, criatividade e trabalho em equipe. Apesar disso, é possível melhorar o torneio através de uma melhor usabilidade da plataforma Robocode, com um design mais moderno e atrativo.

\subsection{Coordenadores e Professores}

Os coordenadores entrevistados validaram os resultados mencionados anteriormente, com uma tendência maior dos participantes do torneio em desenvolver competências ligadas à programação e PC, além de promover o trabalho em equipe na resolução de problemas do robô desenvolvido. O perfil resumido desses entrevistados, além do ano em que participaram da organização do torneio, pode ser visto na Tabela 1. Embora o número amostral de entrevistas seja baixo e limitado ao instituições do estado de São Paulo, essa concentração regional pode indicar problemas na divulgação do torneio em um âmbito nacional para atingir instituições além deste estado. Outra possível explicação é a organização de torneios similares nas outras regiões, o que pode desestimular coordenadores dessas a participarem de um torneio com características diferentes. A dificuldade no levantamento dos contatos dos coordenadores de torneios históricos também pode ter contribuído para essa concentração e baixo número de participantes.

Além dos benefícios já discutidos, outras características pedagógicas foram reportadas. Um dos coordenadores apontou uma grande autonomia dos alunos durante o desenvolvimento do robô, sendo que esses pouco consultavam professores e instrutores com dúvidas técnicas de programação. Esse relato corrobora com a proposta de PBL aplicada na competição, que concede mais independência aos alunos para se desenvolverem e adquirirem novos conhecimentos.

Em termos das atividades dos participantes, todos os coordenadores indicaram que a participação na competição era feita em caráter informal e extracurricular, mesmo que a grade apresentasse matérias ligadas à PC e programação. Somente um coordenador indicou recompensar alunos participantes do torneio com pontuação extra em disciplina de programação, além de permitir que os alunos usassem o tempo em sala de aula para desenvolver seus robôs.

Em termos de participações recorrentes, embora haja uma tendência maior dos coordenadores participarem na organização da competição em anos consecutivos, houve uma notável diminuição desses a partir de 2017. Essa dificuldade, segundo os próprios coordenadores, se deve a uma diminuição no número de alunos interessados na competição. Isso pode ser explicado pelo aumento significativo nos últimos anos de competições científicas análogas ao torneio Robocode, tais como maratonas de programação, hac- 
IX Congresso Brasileiro de Informática na Educação (CBIE 2020)

Anais do XXXI Simpósio Brasileiro de Informática na Educação (SBIE 2020)

Tabela 1. Perfil dos coordenadores de torneio Robocode, por ano de participação

\begin{tabular}{|c|c|c|c|c|c|c|}
\hline \multirow{2}{*}{ Gênero } & \multicolumn{6}{|c|}{ Ano de participação } \\
\cline { 2 - 7 } & 2014 & 2015 & 2016 & 2017 & 2018 & 2019 \\
\hline Masculino & 2 & 5 & 5 & 1 & 2 & 1 \\
\hline Feminino & 1 & 4 & 3 & 0 & 0 & 0 \\
\hline
\end{tabular}

kathons, eventos de empreendedorismo e competições de robótica. Todos os coordenadores entrevistados neste caso reportaram conhecer outras competições similares e estimular seus alunos a participarem de uma gama mais diversificada de competições. Assim, dentre tantas opções possíveis, atrair alunos interessados no torneio têm se tornado uma atividade muito difícil.

Outro ponto de interesse que surgiu durante as entrevistas é a identificação de habilidades que os estudantes levarão para a vida profissional e que estão estreitamente vinculados com o PC e HS21, tais como raciocínio lógico, resolução de problemas, trabalho em equipe, autonomia no seu próprio desenvolvimento pessoal e técnico, e assumir um papel mais ativo no próprio desenvolvimento (também conhecido como protagonismo).

Um relato ilustrativo deste protagonismo e autonomia foi o de uma equipe que após a competição elaborou um conjunto de cartões-guia para auxiliar durante o processo de programação do robô, tendo como inspiração cartões de auxílio da plataforma Scratch; esses cartões-guia mostram um passo a passo em distintos cenários de competição do robô, auxiliando iniciantes em programação a desenvolver um robô mais facilmente.

As características dos coordenadores participantes do torneio Robocode Brasil é diferente da ilustrada pelo coordenador usado como controle. No controle, as competições são usadas como uma forma de engajar de forma lúdica alunos em anos iniciais dos cursos técnico e superior, ligados à Tecnologia da informação (TI), mostrando uma aplicação prática através de exemplos de Robocode e ilustrando atividades de programação usando a movimentação desses robôs. Além disso, a plataforma também é utilizada para demonstrar, claramente, aplicações de conceitos de outras disciplinas, tais como matemática e física. Diferentemente do Robocode Brasil, essa competição e uso da plataforma é feita diretamente em sala de aula. É interessante observar que a aplicação do Robocode neste contexto também é utilizada além dos cursos de TI, tendo o coordenador reportado seu uso em cursos não-técnicos como administração de empresas. Finalmente, o coordenador e a instituição associada a essa competição científica alternativa usam o processo de desenvolvimento do robô como uma forma de identificar líderes entre as equipes, bem como buscar observar traços comportamentais ativamente através de análise dos algoritmos e comportamentos de cada robô desenvolvido. As dificuldades apontadas nesta competição em particular também se diferenciam do Robocode Brasil, pois o maior desafio apontado foi conseguir o apoio de professores nas disciplinas para a disseminação do torneio e seu uso ativo em sala de aula.

Embora existam muitos pontos que possam ser melhorados, foi possível observar através dessas entrevistas que o torneio apresenta valor ao estimular alunos a melhorarem suas competências de PC e estimularem HS21, em especial criatividade e trabalho em equipe. Nesse caso, a competição pode maximizar esses resultados ao considerar formas 
de integrar o desenvolvimento desses robôs em aulas tradicionais, engajar em atividades de divulgação além do contexto do Estado de São Paulo e finalmente estimular alunos que já participaram do torneio a competirem novamente.

\section{Conclusões}

O trabalho ilustra os resultados da aplicação de uma competição científica, no âmbito nacional, que utiliza a plataforma Robocode, um jogo sério cujo objetivo é o ensino de competências de programação através da linguagem Java. Neste contexto, alunos e exalunos de ensino médio e superior de diversas instituições participantes entre os anos de 2014 e 2019 foram entrevistados, juntamente com coordenadores dessas competições em cada instituição. Ao todo foram registrados experiências de 68 participantes e 13 coordenadores, que reportaram um estímulo na aplicação das competências de PC, como algoritmos/programação além de modelagem e abstração de problema. Além disso, foram reportadas o uso de habilidades como o trabalho em equipe, criatividade, resolução de problemas e gestão de projetos. $\mathrm{O}$ torneio ainda estimulou participantes a seguir carreiras nas áreas de STEM e gestão de equipes.

Embora os resultados indiquem experiências positivas no aprendizado de PC e HS21, esses foram considerados somente no contexto de uma competição particular e seus benefícios podem variar conforme as regras e contexto de outras competições. Além disso, o número de entrevistados foi baixo quando comparado ao número total de participantes do torneio e muito localizado em instituições de ensino no Estado de São Paulo. Trabalhos futuros podem analisar o contexto desta competição em outros estados, bem como observar como a plataforma Robocode têm sido aplicada em outras competições, no contexto de sala de aula, ou mesmo outros jogos sérios em ambientes competitivos para ensino de STEM.

\section{Agradecimentos}

O presente trabalho foi realizado com apoio da Coordenação de Aperfeiçoamento de Pessoal de Nível Superior - Brasil (CAPES) - Código de Financiamento 001.

\section{Referências}

Alhaddab, T. A. and Alnatheer, S. A. (2015). Future scientists: How women's and minorities' math self-efficacy and science perception affect their stem major selection. In IEEE Integrated STEM Education Conference. IEEE.

Ali, M., Talib, C. A., Surif, J., Ibrahim, N. H., and Abdullah, A. H. (2018). Effect of stem competition on stem career interest. In IEEE 10th International Conference on Engineering Education - ICEED. IEEE.

Almeida, F., Faria, D., and Queirós, A. (2017). Strengths and limitations of qualitative and quantitative research methods. European Journal of Education Studies, 3:369-387.

Amaral, L., Silva, G. B. e., and Pantaleão, E. (2015). Plataforma robocode como ferramenta lúdica de ensino de programação de computadores - pesquisa e extensão universitária em escolas públicas de minas gerais. 26(1):200.

Boyle, E. A., Hainey, T., Connolly, T. M., Gray, G., Earp, J., Ott, M., Lim, T., Ninaus, M., Ribeiro, C., and Pereira, J. (2016). An update to the systematic literature review 
IX Congresso Brasileiro de Informática na Educação (CBIE 2020)

Anais do XXXI Simpósio Brasileiro de Informática na Educação (SBIE 2020)

of empirical evidence of the impacts and outcomes of computer games and serious games. Computers \& Education, 94:178-192.

Brennan, K. and Resnick, M. (2012). New frameworks for studying and assessing the development of computational thinking. In The American Educational Research Association - AERA, pages 1-25. AERA.

ComputerWorld (2019). Estas são as 10 linguagens de programação mais utilizadas em 2019. URL: https://computerworld.com.br/2019/11/08/estas-sao-as-10-linguagens-deprogramacao-mais-utilizadas-em-2019/ [Last access on July, 2020].

J., K. and Gibson, J. (2006). Robocode problem-based learning: A non-prescriptive approach to teaching programming. ACM SIGCSE Bulletin, 38(3):217-221.

Leydens, J. A., Moskal, B. M., and Pavelich, M. J. (2004). Qualitative methods used in the assessment of engineering education. Journal of Engineering Education, 93(1):65-72.

LIAG - UNICAMP (2020). Torneio robocode. URL: https://liag.ft.unicamp.br/robocode/ [Last access on June, 2020].

Meira, M. C. (2016). Aprendizagem de linguagem de programação com metodologia PBL em competições científicas com Robocode = Learning programming language with problem based learning methodology in scientific competitions with Robocode. Dissertação (mestrado), Universidade Estadual de Campinas, Faculdade de Tecnologia, Limeira, SP.

Moust, J., Bouhuijs, P., and Schmidt, H. (2016). Introduction to Problem-Based Learning. A guide for students. Routledge, 6th edition.

Seemiller, C. and Grace, M. (2017). Generation z: Educating and engaging the next generation of students. About Campus, 22(3):21-26.

Shatto, B. and Erwin, K. (2016). Moving on from millennials: Preparing for generation z. The Journal of Continuing Education in Nursing, 47(6):253-254.

Shute, V. J., Sun, C., and Asbell-Clarke, J. (2017). Demystifying computational thinking. Educational Research Review, 22:142-158.

Sousa, C. and Costa, C. (2018). Videogames as a learning tool: Is game-based learning more effective? Revista Lusófona de Educação, 40:199-240.

Starman, A. B. (2013). The case study as a type of qualitative research. Journal of Contemporary Educational Studies, 64(1):28-43.

Weintrop, D. and Wilensky, U. (2012). Robobuilder: A program-to-play constructionist video game. In Proceedings of the Constructionism Conference.

Wing, J. M. (2006). Computational thinking. Communications of the ACM, 49(3):33-158.

Wing, J. M. (2008). Computational thinking and thinking about computing. Philosophical Transactions of the Royal Society A: Mathematical, Physical and Engineering Sciences, 366(1881):3717-3725.

World Economic Forum (2018). The future of jobs report - insight report. URL: https://www.voced.edu.au/content/ngv:80780 [Last access on June, 2020].

Yew, E. H. J. and Goh, K. (2016). Problem-based learning: An overview of its process and impact on learning. Health Professions Education, 2(2):75-79. 